\title{
Influence of the thalamus on spatial visual processing in frontal cortex
}

\author{
Marc A. Sommer ${ }^{1,2} \&$ Robert H. Wurtz ${ }^{2}$
}

Each of our movements activates our own sensory receptors, and therefore keeping track of self-movement is a necessary part of analysing sensory input. One way in which the brain keeps track of self-movement is by monitoring an internal copy, or corollary discharge, of motor commands ${ }^{1-13}$. This concept could explain why we perceive a stable visual world despite our frequent quick, or saccadic, eye movements: corollary discharge about each saccade would permit the visual system to ignore saccade-induced visual changes ${ }^{6-9}$. The critical missing link has been the connection between corollary discharge and visual processing. Here we show that such a link is formed by a corollary discharge from the thalamus that targets the frontal cortex. In the thalamus, neurons in the mediodorsal nucleus relay a corollary discharge of saccades from the midbrain superior colliculus to the cortical frontal eye field ${ }^{10-12}$. In the frontal eye field, neurons use corollary discharge to shift their visual receptive fields spatially before saccades ${ }^{14,15}$. We tested the hypothesis that these two components-a pathway for corollary discharge and neurons with shifting receptive fields-form a circuit in which the corollary discharge drives the shift. First we showed that the known spatial and temporal properties of the corollary discharge predict the dynamic changes in spatial visual processing of cortical neurons when saccades are made. Then we moved from this correlation to causation by isolating single cortical neurons and showing that their spatial visual processing is impaired when corollary discharge from the thalamus is interrupted. Thus the visual processing of frontal neurons is spatiotemporally matched with, and functionally dependent on, corollary discharge input from the thalamus. These experiments establish the first link between corollary discharge and visual processing, delineate a brain circuit that is well suited for mediating visual stability, and provide a framework for studying corollary discharge in other sensory systems.

The dominant hypothesis of how we perceive visual stability is that advance warning of saccades is sent to the visual system (Supplementary Fig. S1a, b) ${ }^{7-9}$. The only known pathway for saccadic corollary discharge innervates the cortical frontal eye field (FEF) ${ }^{10-12}$ (Supplementary Fig. S1c), which contains visually responsive neurons that have been shown ${ }^{14,15}$ to use corollary discharge. Neurons of this type, which were discovered in the parietal cortex ${ }^{16}$, alter their spatial visual processing just before a saccade (Fig. 1a). Specifically, they shift their visual receptive field (RF) to a new location, the future field (FF), where the RF will reside after the saccade ${ }^{14-18}$. Because the neurons sample the same region of visual space before and after the saccade (in the FF and postsaccadic RF, respectively), they provide information about whether a visual scene is stable across saccades.

We trained monkeys to make saccades while a visual probe appeared at the RF or FF during initial fixation or just before a saccade (Fig. 1b; Supplementary Fig. S2). An example neuron had a visual response in its RF, but not in its FF, during initial fixation (Fig. 1c, left column), but this spatial sensitivity reversed just before a saccade: the FF became responsive and the RF unresponsive (Fig. 1c, middle column). Saccades alone evoked no activity as revealed in trials without a probe (Fig. 1c, right column, bottom row). Activity in the FF thus reflected a sudden, presaccadic onset of visual sensitivity-a shifting RF.

We searched in the FEFs of two monkeys for neurons with shifting RFs. Only neurons identified as belonging to the superior colliculus (SC)-FEF circuit as defined by antidromic and orthodromic stimulation criteria were studied (antidromic and orthodromic neurons a

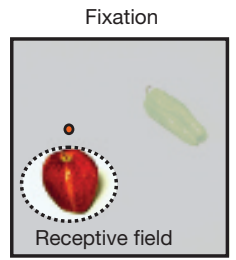

b$$
\text { (1) }
$$
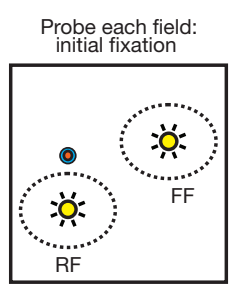

c

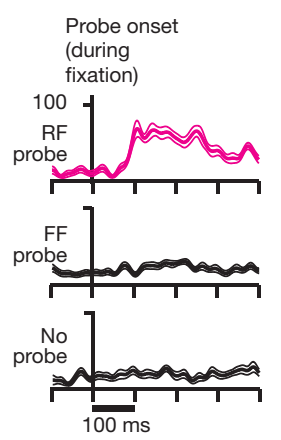

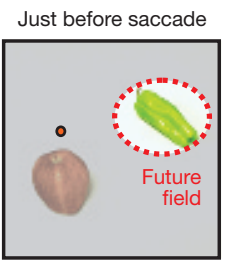

Probe each field:

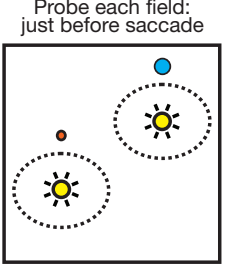

Probe onset (just before saccade)
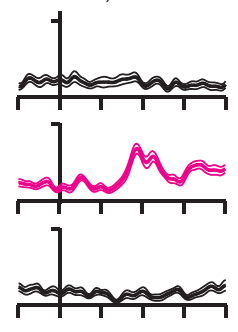

Saccade

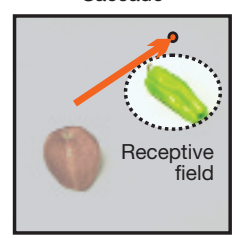

$4^{\circ}$

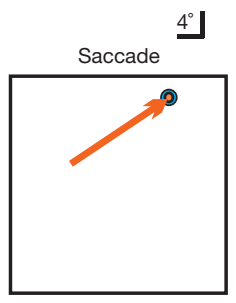

Saccade initiation

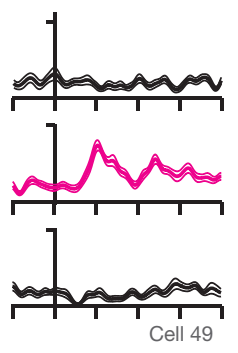

Figure 1 | Shifting receptive fields of visual neurons. a, Just before a saccade, visual responsiveness moves from the RF to the FF. $\mathbf{b}$, Monkeys looked at (orange dot) a fixation spot (blue dot, left) and made a saccade to a target (blue dot, middle). We flashed a probe (yellow circle) in one of two locations and two times (one probe per trial). c, Example FEF neuron. The firing rate (mean \pm s.e.m.; the vertical scale is in spikes $s^{-1}$ ) is aligned with events represented in $\mathbf{a}$ and $\mathbf{b}$ above. The visual response shifts from the RF (magenta, left) to the FF (magenta, middle) just before the saccade. 
behaved identically and were pooled; see Supplementary Notes). Of 71 such neurons that had visual responses, $61 \%(n=43)$ had shifting RFs. One-third (14 of 43) showed a complete shift from RF to FF just before saccade initiation (for example Fig. 1c), whereas the rest (29 of 43) became active at the FF while maintaining responsiveness at the RF (Supplementary Fig. S3). The basic properties of the neurons with shifting RFs were similar to those described previously ${ }^{14-18}$.

If FEF neurons with shifting RFs use corollary discharge from the SC, the shifts should be influenced by the spatiotemporal dynamics of SC activity. Spatially, SC activity encodes saccades on a topographic map ${ }^{19}$. Localized activity on the map specifies the vector (eccentricity and direction) of the saccadic target (Fig. 2a, 'hill' of activity). Temporally, SC activity encodes the time of saccade initiation with a volley of action potentials (Fig. 2a, arrow representing sudden onset). Thus we predicted that, spatially, shifting RFs should jump as if directed by a vector specification of the saccade, and temporally, they should move in synchrony with the saccade as if driven by a burst of presaccadic activity.

To study the spatial properties of the shift, we added a visual probe at the midpoint between the RF and the FF. If activity at the midpoint were unchanged before a saccade, this would show that visual sensitivity 'jumped' from the RF to the FF (Fig. 2b, top). However, if activity at the midpoint increased before a saccade, this would refute the prediction of a jump and suggest that a gradual spread of visual
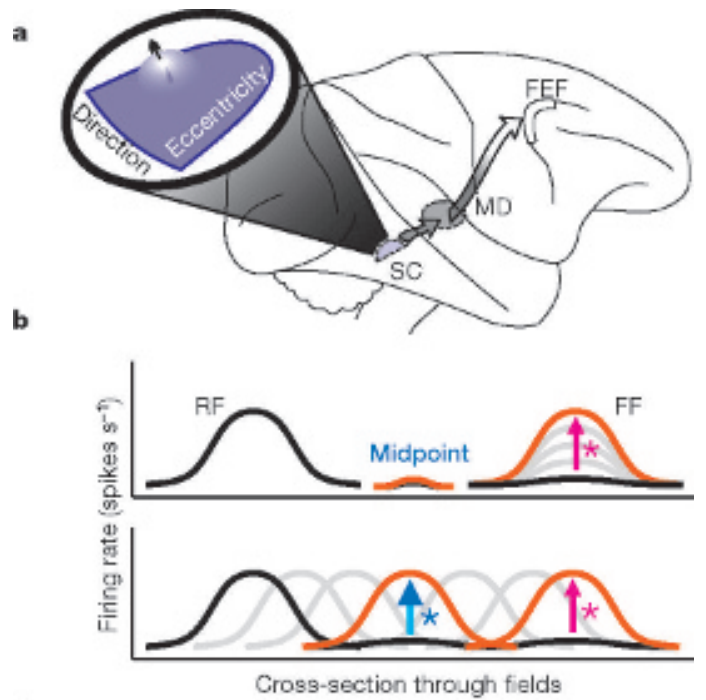

c

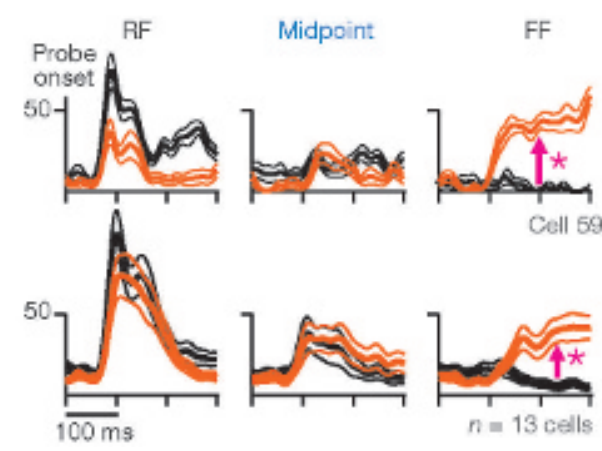

Figure 2 | The spatial properties of shifting RFs are predicted by corollary discharge from the SC-MD-FEF pathway. a, The corollary discharge arises from the SC, which encodes saccades spatially, using a map of direction and eccentricity, and temporally, using a burst of activity. b, Spatially, we predicted that shifting RFs jump (top), as indicated by a significant (asterisk) increase in activity at the FF (magenta arrow) but no significant difference at the midpoint. Alternatively, shifting RFs could spread (bottom). Probe onset time: black, initial fixation; orange, just before saccade. c, Example neuron (top) and population (bottom); data aligned to probe onset. Shifting RFs moved as a jump. The vertical scale is in spikes $\mathrm{s}^{-1}$. sensitivity travelled from the RF to the FF (Fig. 2b, bottom). An example neuron responded to a probe in the RF during initial fixation (Fig. 2c, top) but had little if any response at the midpoint and was unresponsive at the FF. Just before the saccade, the neuron suddenly became visually responsive at the FF $(P<0.0001)$, whereas the midpoint location showed no significant change $(P>0.05$; measured 100-300 ms after probe onset). We isolated 13 neurons long enough to test the midpoint, and every one exhibited no change there as activity jumped to the FF. The pooled data from all 13 neurons (Fig. 2c, bottom) showed that just before a saccade, the average activity dropped slightly at the RF, rose markedly at the FF (by 43 spikes $\left.\mathrm{s}^{-1}, P<0.0001\right)$ and did not change significantly at the midpoint $(P>0.05)$. A minor, late increase at the midpoint was due to a slight overlap of the probe onto the edge of the FF (see further analyses in Supplementary Figs S4-S6). Shifting RFs therefore jump, rather than spread, to their new locations.

Next we tested our prediction that shifts were synchronized to saccade initiation by flashing probes earlier to impose a substantial delay (about $200 \mathrm{~ms}$ ) between probe onset and saccade initiation. If shifts were synchronized to saccade initiation they would start after the delay, when the saccades begin, but if shifts were synchronized to probe onset they would start at a normal visual latency after the probes. On a trial-by-trial basis, we found that the times of shift onset and saccade initiation were tightly correlated in single neurons (Fig. 3a, left; $R=0.97, P<0.001$ ) and in the population (Fig. 3b; mean $R=0.50$, greater than 0 at $P<0.002$, $t$-test; $n=13$ neurons having more than ten trials). In addition, there was a higher peak in the average firing rate when aligned to saccade initiation (Fig. 3a, right) as opposed to probe onset (Fig. 3a, left; true for 24 of 26 individual neurons, an average increase of 6 spikes s $^{-1} ; P=0.001$, paired $t$-test). In contrast, shift onset had no clear relation to probe onset; the shift started long after the normal visual latency (green arrow) in the single example (Fig. 3a, left) and in the population (not shown; average shift onset time $174 \pm 92 \mathrm{~ms}$ (mean \pm s.d.) versus average visual latency $86 \pm 16 \mathrm{~ms} ; P<0.0001)$. In sum, shifting RFs were synchronized with saccades. On average they started with saccade initiation (Fig. 3c; mean $24 \pm 74$ ms (mean \pm s.d.),
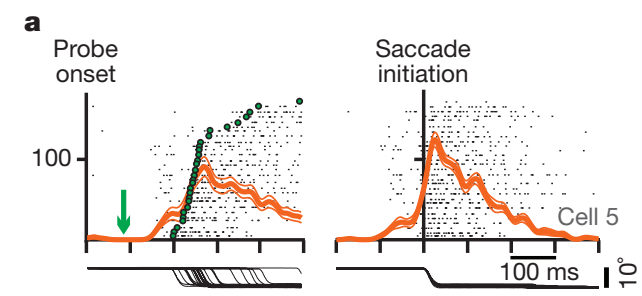

b
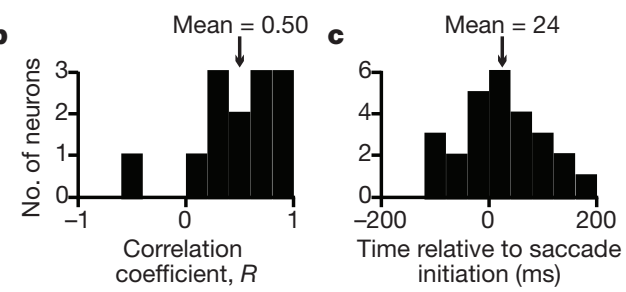

Figure 3 | The temporal properties of shifting RFs are predicted by corollary discharge from the SC-MD-FEF pathway. a, Our hypothesis predicts that shifting RFs are synchronized with saccades. Shift activity (in the FF) for an example neuron is aligned to probe onset (left) and saccade initiation (right). Rasters of action potentials from each trial are sorted by saccadic latency (green dots). Eye position traces (horizontal component) are shown below. The green arrow indicates the average visual latency of this neuron. The vertical scale is in spikes $\mathrm{s}^{-1} \cdot \mathbf{b}$, Strength of correlation (Pearson's $R$ ) between shift onset time and saccadic latency for the population. Mean $R>0$ at $P<0.002$. c, Shift onset time relative to saccade initiation for the population. There is no significant difference in shift onset time from $0 \mathrm{~ms}$. 
not significantly different from 0 ; range -94 to $164 \mathrm{~ms}$ ). A visual response gated by saccadic initiation would be critical for perceiving visual stability, because saccades can be cancelled about $100 \mathrm{~ms}$ before initiation ${ }^{20}$; only after this 'point of no return', when a saccade is inevitable, should neurons shift their RFs.

Next we looked for evidence that corollary discharge from SC through the thalamus caused the shifting RFs in the FEF. In an experimental session, first we isolated a visually responsive FEF neuron that was connected to the SC and had a shifting RF (as described for the 43 neurons above). While maintaining isolation of the neuron, we inserted an injection syringe needle targeted at the previously identified relay nucleus from the SC to the FEF in the mediodorsal (MD) thalamus (Fig. 4a). We then returned to the FEF neuron, quantified its visual sensitivity at the RF and the FF, and inactivated the MD nucleus with muscimol, an agonist of the inhibitory neurotransmitter $\gamma$-aminobutyric acid (GABA) ${ }^{21}$. Finally, we retested the visual sensitivity of the same neuron at the RF and the FF. Our prediction was that MD inactivation should reduce activity at the FF.

We completed the lengthy sequence of procedures required in this experiment for eight FEF neurons (Supplementary Fig. S7). In an example neuron, the major effect was a $70 \%$ decrease $(P<0.0001)$ in FF activity (Fig. 4b, lower middle and right), whereas the visual response in the RF was spared (Fig. 4b, upper left), as was low-level
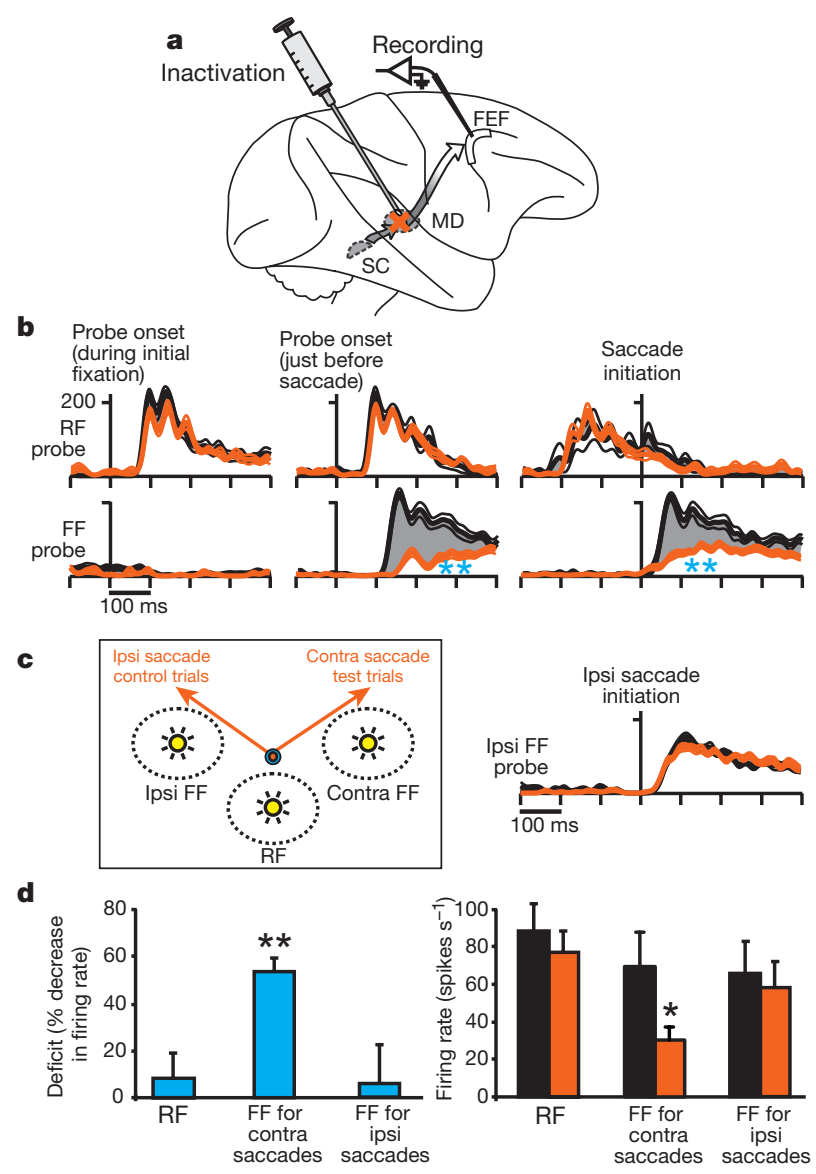

Figure 4 | Shifting RFs require corollary discharge from the pathway. a, We recorded from FEF neurons while inactivating MD relay neurons that convey corollary discharge. $\mathbf{b}$, Example neuron (the same one as in Supplementary Fig. S3), with the difference in activity between during inactivation (orange) and before inactivation (black) highlighted in grey. Visual responsiveness in the FF just before the saccade- the shiftdecreased severely during MD inactivation. The vertical scale is in spikes s ${ }^{-1}$. c, In contrast, in ipsiversive (ipsi) saccade trials for the example neuron (left) there was no deficit (right). Contra, contraversive. d, Population data. Left, average percentage deficits; right, average changes in raw visual response (means and s.e.m.). Asterisk, $P<0.05$; two asterisks, $P<0.0001$. activity in the trials without a probe (not shown). Inactivating the corollary discharge pathway through the MD therefore caused a marked reduction in the ability of this neuron to shift its RF.

Because the SC-MD-FEF pathway on each side of the brain represents only contraversive saccades ${ }^{10}$, a further prediction was that our unilateral inactivations would eliminate corollary discharge for contraversive saccades only. Therefore shifts accompanying contraversive saccades, but not ipsiversive saccades, should be impaired. This is exactly what we found (Fig. 4c).

Overall, we found that activity at the FF decreased during inactivation for every neuron (16-70\%, individually significant for seven of eight neurons; Supplementary Fig. S7). The average deficit (Fig. 4d, left) was severe in the FF for contraversive saccades (53.9\% deficit, $P<0.001)$ but not significant in the RF $(P=0.48)$ or in the FF for ipsiversive saccades $(P=0.76)$. In terms of firing rates (Fig. $4 \mathrm{~d}$, right), the average visual response decreased from 69.6 to 30.3 spikes s $^{-1}$ in the FF for contraversive saccades (paired $t$-test, $P=0.019$ ) but was unchanged in the RF $(P=0.2)$ and in the FF for ipsiversive saccades $(P=0.4)$. Data from the trials without a probe never showed a significant deficit (not shown). Most of the neurons were antidromically activated from the SC, but the one orthodromically activated neuron showed the same effect (55\% deficit; Supplementary Fig. S7a). Injections as small as $0.4 \mu \mathrm{l}$ were effective and larger volumes did not cause larger effects, suggesting that inactivation beyond the targeted MD relay neurons was inconsequential (Table in Supplementary Fig. S7). A side-product of MD inactivation, bilaterally increased saccadic latencies, did not cause the deficits (Supplementary Fig. S8). For more consideration of these issues see the Supplementary Discussion.

We conclude that corollary discharge from the SC-MD-FEF pathway is both appropriate and necessary to produce shifting RFs in FEF neurons. These results delineate a link between an identified corollary discharge and a sensory system in the primate brain. More specifically, the data provide a circuit-level explanation for our percept of visual stability across saccades - an aim of neuroscience for at least 50 years $^{1,8,9}$. Corollary discharge from the SC causes the visual system to shift its RFs spatially, and these shifting RFs are proposed to promote the percept of visual stability across saccades ${ }^{16}$ (although exactly how this would be achieved has not yet been modelled).

Our findings permit the first direct test of the visual-stability hypothesis. In monkeys trained to detect visual motion, one could inactivate MD relay neurons to reduce shifting RFs; if these shifts underlie perceptual stability, the monkeys should report that static visual stimuli move with saccades. More generally, our approach may open up the study of other corollary discharge circuits such as those mediating the percept of a stable soundscape during head movements.

\section{METHODS}

Two rhesus monkeys (Macaca mulatta), 'T' and ' $\mathrm{Y}$ ', were prepared for neurophysiological experiments as described in detail elsewhere ${ }^{10,22}$. Details on physiology, task design and analysis are given in Supplementary Methods. To record from FEF neurons, we used standard extracellular methods combined with antidromic and orthodromic stimulation ${ }^{10,22}$ to confirm a connection with the SC (Supplementary Notes). To inactivate MD relay neurons, we first mapped their location in the thalamus by activating each one antidromically from the FEF and orthodromically from the SC. Relay neurons of the SC-MD-FEF pathway were concentrated in a restricted volume along the lateral edge of the MD thalamus ${ }^{10,23}$. We inactivated them by inserting the tip of a 30 -gauge cannula at their location and injecting $5 \mu \mathrm{g} \mathrm{\mu l}^{-1}$ muscimol.

Received 29 June; accepted 25 September 2006.

Published online 8 November 2006.

1. Teuber, H.-L. in The Frontal Granular Cortex and Behavior (eds Warren, J. P. \& Akerts, K.) 410-444 (McGraw-Hill, New York, 1964).

2. Delcomyn, F. Corollary discharge to cockroach giant interneurons. Nature 269, 160-162 (1977).

3. Poulet, J. F. \& Hedwig, B. A corollary discharge maintains auditory sensitivity during sound production. Nature 418, 872-876 (2002). 
4. Bell, C. C. in Comparative Physiology: Sensory Systems (eds Bolis, L. \& Keynes, R. D.) 637-646 (Cambridge Univ. Press, Cambridge, 1984).

5. Thier, P., Haarmeier, T., Chakraborty, S., Lindner, A. \& Tikhonov, A. Cortical substrates of perceptual stability during eye movements. Neuroimage 14, S33-S39 (2001).

6. Wurtz, R. H. \& Sommer, M. A. Identifying corollary discharges for movement in the primate brain. Prog. Brain Res. 144, 47-60 (2004).

7. Von Helmholtz, H. Helmholtz's Treatise on Physiological Optics (Thoemmes, Bristol, 2000).

8. Sperry, R. W. Neural basis of the spontaneous optokinetic response produced by visual inversion. J. Comp. Physiol. Psychol. 43, 482-489 (1950).

9. Von Holst, E. \& Mittelstaedt, H. Das Reafferenzprinzip. Wechselwirkungen zwischen Zentralnervensystem und Peripherie. Naturwissenschaften 37, 464-476 (1950).

10. Sommer, M. A. \& Wurtz, R. H. What the brain stem tells the frontal cortex. I. Oculomotor signals sent from superior colliculus to frontal eye field via mediodorsal thalamus. J. Neurophysiol. 91, 1381-1402 (2004).

11. Sommer, M. A.\& Wurtz, R. H. What the brain stem tells the frontal cortex. II. Role of the SC-MD-FEF pathway in corollary discharge. J. Neurophysiol. 91, 1403-1423 (2004).

12. Sommer, M. A. \& Wurtz, R. H. A pathway in primate brain for internal monitoring of movements. Science 296, 1480-1482 (2002).

13. Guthrie, B. L., Porter, J. D. \& Sparks, D. L. Corollary discharge provides accurate eye position information to the oculomotor system. Science 221, 1193-1195 (1983).

14. Umeno, M. M. \& Goldberg, M. E. Spatial processing in the monkey frontal eye field. I. Predictive visual responses. J. Neurophysiol. 78, 1373-1383 (1997).

15. Umeno, M. M. \& Goldberg, M. E. Spatial processing in the monkey frontal eye field. II. Memory responses. J. Neurophysiol. 86, 2344-2352 (2001).

16. Duhamel, J. R., Colby, C. L. \& Goldberg, M. E. The updating of the representation of visual space in parietal cortex by intended eye movements. Science 255, 90-92 (1992).
17. Kusunoki, M. \& Goldberg, M. E. The time course of perisaccadic receptive field shifts in the lateral intraparietal area of the monkey. J. Neurophysiol. 89, 1519-1527 (2003).

18. Nakamura, K. \& Colby, C. L. Updating of the visual representation in monkey striate and extrastriate cortex during saccades. Proc. Natl Acad. Sci. USA 99, 4026-4031 (2002).

19. Sparks, D. L. \& Hartwich-Young, R. in The Neurobiology of Saccadic Eye Movements; Reviews of Oculomotor Research Vol. III (eds Wurtz, R. H. \& Goldberg, M. E.) 213-255 (Elsevier, Amsterdam, 1989).

20. Hanes, D. P. \& Schall, J. D. Countermanding saccades in macaque. Vis. Neurosci. 12, 929-937 (1995).

21. Lomber, S. G. The advantages and limitations of permanent or reversible deactivation techniques in the assessment of neural function. J. Neurosci. Methods 86, 109-117 (1999).

22. Sommer, M. A. \& Wurtz, R. H. Composition and topographic organization of signals sent from the frontal eye field to the superior colliculus. J. Neurophysiol. 83, 1979-2001 (2000)

23. Lynch, J. C., Hoover, J. E. \& Strick, P. L. Input to the primate frontal eye field from the substantia nigra, superior colliculus, and dentate nucleus demonstrated by transneuronal transport. Exp. Brain Res. 100, 181-186 (1994).

Supplementary Information is linked to the online version of the paper at www.nature.com/nature.

Acknowledgements This research was supported by the Intramural Research Program of the NIH and the NEI.

Author Contributions Both authors designed the experiments, M.A.S. performed and analysed them, and both authors wrote the paper.

Author Information Reprints and permissions information is available at www.nature.com/reprints. The authors declare no competing financial interests. Correspondence and requests for materials should be addressed to M.A.S (sommer@bns.pitt.edu). 


\section{Supplementary Information}

\section{Supplementary Methods}

During an initial aseptic surgery, we implanted chambers over the SC, MD, and FEF, plus a post for restraining the head during experiments and scleral search coils for measuring eye position. Filtering of the eye position signal introduced negligible time lags in determination of saccadic onset ( 1 ms maximally). The continuous eye position records were sampled at $1 \mathrm{kHz}$ for offline analysis. Action potentials were recorded at 1 ms resolution.

Monkeys were trained to fixate a spot and make a saccade to a target placed well outside of the neuron's RF (Supplementary Fig. S2). Meanwhile, task-irrelevant visual probes were flashed to quantify visual sensitivity at the RF and FF. A monkey faced a tangent screen onto which visual stimuli were back-projected with an LCD projector. The configuration of probes and saccade targets was tailored to each neuron according to the following criteria. First, the classical response field was mapped using visuallyguided saccade tasks at multiple directions and amplitudes. The RF probe stimulus location was at the middle of this classical response field. Second, we chose a saccade (sacc1 in Supplementary Fig. S2) that resulted in a FF probe stimulus location completely outside of the response field. During initial fixation, therefore, probes at the FF location failed to evoke visual responses. Because sacc1 went away from the RF, any saccaderelated activity of the neuron was minimized or eliminated. Third, the sacc1 had to be contraversive, at least in the inactivation studies, due to the contraversive laterality of the SC-MD-FEF pathway. Once an arrangement was found that involved a contraversive 
sacc1 that kept the FF location out of the RF during initial fixation, an ipsiversive control sacc1 was selected as the mirror image.

The sacc1 was followed by a fixation period and then the generation of a second saccade (sacc2 in Supplementary Fig. S2) directed away from the FF. The purpose of sacc2 was to provide a subsequent saccadic target after sacc1 to explicitly prevent any saccades to the location of the probe ${ }^{14}$. Saccadic targets were $0.1 \mathrm{deg}$. wide red squares and visual probes were 0.2 deg. wide white squares (for illustration purposes they are depicted as blue and yellow, respectively, in the Figures). Monkeys were trained to look at the saccadic targets, and in the rare trials in which they made a saccade to a probe ( $<1 \%$ of trials), the trial was aborted.

Temporally, we flashed probes either during initial fixation or, on separate trials, just before the saccade. To achieve the latter timing despite the unpredictable nature of saccadic latencies, we illuminated the probe $\sim 120 \mathrm{~ms}$ after onset of the saccadic target. Saccadic latencies had a mean of about $190 \mathrm{~ms}$, and thus the probe would appear and disappear 50 ms later just before initiation of about 2/3 of saccades (we discarded the other $1 / 3$ of trials in which saccade initiation occurred while the probe was still lit; the number of remaining trials per condition was typically 8-20). Probe timings were measured precisely using a photodiode. To achieve probe onset during initial fixation, we illuminated the probe 200 ms after the monkey acquired the fixation spot (about 390$590 \mathrm{~ms}$ before the saccade). No-probe trials were implemented by setting the probe color and luminance to that of the background. Finally, for some neurons we implemented a special probe timing to temporally dissociate probe onset and saccade initiation (see Fig. 3). To do this, on randomly interleaved trials the probe appeared simultaneously with the saccadic target (probe “ $x$ ” in Supplementary Fig. S2). 
Specific analyses are described in the text. In general, action potential data were analyzed by calculating the average firing rates from spike rasters in 100 ms epochs. A visual response was considered significant if the activity during an analysis epoch was greater in probe trials than in the same epoch in time-matched, no-probe trials. The analysis epoch for measuring an RF response spanned 50-150 ms after probe onset. The analysis epoch for measuring an FF response varied because of the diverse shift latencies (see Fig. 3c). Because we found that the shifts were synchronized to saccade initiation, analysis of FF activity was aligned to saccade initiation. The earliest epoch began $-60 \mathrm{~ms}$ before saccadic initiation for the most presaccadic shifts (e.g. Supplementary Fig. S7b) and the latest epoch began 160 ms after saccadic initiation for longest-latency shifts (e.g. Supplementary Fig. S7c). In the inactivation experiments, identical epochs were used to analyze the before and during inactivation data.

To analyze shift onset latencies we used a burst detection method ${ }^{24}$ to calculate the start of the shift on a trial-by-trial basis for correlation with saccadic latency (Fig. 3b). We eliminated any neurons with saccade-related activity as seen in no-probe trials to ensure that the trial-by-trial rasters of activity reflected the visual response and not any underlying motor discharge.

Throughout the study we statistically analyzed the data using Student's t-test and paired t-test, Pearson's correlation test (Spearman's test gave the same results), and similar methods as noted in the text, using a p level of 0.05 for significance (corrected for multiple comparisons if necessary).

\section{Supplementary Notes}

The FEF neurons included in this study were not just randomly encountered but were physiologically identified as either antidromically activated (back-fired) or 
orthodromically activated (synaptically driven) from the SC. Of our 43 neurons with shifting RFs, 35 were antidromically and 8 orthodromically activated. We examined only physiologically identified neurons for the following reasons. First, from a theoretical standpoint a randomly encountered neuron in the FEF may be connected with many possible structures, but we were studying the influence of the SC on the FEF. Hence, it was reasonable to limit our work to neurons connected with the SC. Second, examining only SC-connected neurons provided many technical benefits: (1) From previous work ${ }^{10}$ we knew that orthodromically activated neurons were highly visual, so finding an orthodromically activated neuron essentially guaranteed its visual nature. Ideally, we would have limited our sample to orthodromically activated neurons (since they are the immediate targets of the SC-MD-FEF pathway). This was not feasible, however, because they seem to be small, layer IV cells ${ }^{10,25}$ and it is difficult to keep one isolated for more than an hour. (2) From past experience we also knew that antidromically activated neurons - which are layer $\mathrm{V}$ pyramidal cells ${ }^{22,26}$ - are very stable during prolonged recordings. Hence the majority of our neurons were antidromically activated, for this technical reason. That our results were robust for these neurons indicates that corollary discharge from the SC permeates through the FEF, not only affecting the recipient layer IV but also output layer V. (3) Activating a neuron from the SC provided us with further parameters for confirming that the neuron was perfectly isolated throughout the long experiments. We cannot overstate the importance of this last point. Besides inspecting the waveform as evidence for continued isolation, we repeatedly checked to see if the neuron was activated or not, and whether the activation latency and current threshold stayed constant. The ability to activate a neuron, and its activation parameters, are stable 
for individual neurons but vary considerably between neighboring neurons in the FEF. Moreover, use of the collision test ${ }^{27}$ further helped us confirm perfect isolation for antidromically activated neurons. A neuron only passes the collision test on $100 \%$ of trials if it is perfectly isolated (another neuron intruding on the isolation will fail to collide the activated neuron)

24. Hanes, D. P., Thompson, K. G. \& Schall, J. D. Relationship of presaccadic activity in frontal eye field and supplementary eye field to saccade initiation in macaque: Poisson spike train analysis. Exp. Brain Res. 103, 85-96 (1995).

25. Shin, S. \& Sommer, M. A. Frontal eye field input neurons have higher spontaneous firing rates and narrower action potentials than output neurons. Program No. 138.11/D59, 2006 Abstract Viewer/Itinerary Planner, Atlanta, GA: Society for Neuroscience

26. Fries, W. Cortical projections to the superior colliculus in the macaque monkey: a retrograde study using horseradish peroxidase. J. Comp. Neurol. 230, 55-76 (1984).

27. Lemon, R. IBRO Handbook Series: Methods in the Neurosciences. Vol. 4. (J. Wiley \& Sons, New York, 1984).

28. Walker, M. F., Fitzgibbon, E. J. \& Goldberg, M. E. Neurons in the monkey superior colliculus predict the visual result of impending saccadic eye movements. J. Neurophysiol. 73, 1988-2003 (1995).

29. Guillery, R. W. \& Sherman, S. M. The thalamus as a monitor of motor outputs. Philos. Trans. R. Soc. Lond. B Biol. Sci. 357, 1809-1821 (2002). 
30. A. S. Tolias et al. Eye movements modulate visual receptive fields of V4 neurons. Neuron 29, 757-767 (2001).

\section{Supplementary Discussion}

Our study showed that the corollary discharge is appropriate and necessary for causing the shift. An obvious follow-up question is whether it is sufficient. We can think of two approaches to test whether the corollary discharge is sufficient to cause the shift, but neither seems feasible. First, one could electrically stimulate MD relay neurons to induce a corollary discharge signal. Perhaps this, by itself, would cause RFs to shift. However, MD relay neurons are tightly packed with no known topography, and they lie very near corticofugal fibers. Electrical stimulation in MD therefore would have unpredictable effects and would likely activate both descending and ascending pathways. Second, one could eliminate all sources of corollary discharge to the FEF except for the SC-MD-FEF pathway. Since we do not know of any other such sources, this cannot be done.

Three alternative explanations for the inactivation deficits are important to discuss. First, MD inactivation causes a slight lengthening of saccadic reaction time bilaterally that may reflect a general decrease in arousal or attentiveness ${ }^{11}$. It could be that this effect contributed to the impaired shifts. Because our shift deficits were unilateral, however, on the face of it we thought it was very unlikely that increased reaction times could have affected our results.

Nevertheless, to be rigorous we carefully examined whether increased reaction time during inactivation may have somehow resulted in a trivial decrease in visual responsiveness at the FF. Recall that to present a probe "just before the saccade", we 
flashed it at a set time following onset of the saccadic target (Supplementary Fig. S2). Therefore, reaction times that increased during the course of an experimental session would increase the delay between appearance of the probe and initiation of the saccade. The FF visual response was synchronized to saccade initiation, and thus longer reaction times and the resulting longer probe-saccade delays would lead to later FF visual responses. This, in principle, could lead to dissipation of the FF visual response.

To test this possibility, we pooled together all of the individual trials from every neuron (contraversive saccade trials only). Plotted in Supplementary Figure S8 is the strength of the FF shift in each trial as a function of probe-saccade delay (PSD). "Before” data are shown with black o symbols, and "during” data with orange x symbols. Because the absolute firing rates of FF visual responses varied considerably between neurons, we normalized the activity in each trial to the average FF visual response for that neuron in the baseline state (before inactivation). For example, consider the data point indicated by the blue arrow, at coordinate $(\mathrm{PSD}=350$, norm. firing rate $=0.37)$. In this trial, a probe was flashed in the FF of the neuron with the intention that the probe would appear just before saccade initiation. However, because the saccadic reaction time was long, there was actually a delay of $350 \mathrm{~ms}$ between the probe onset and the saccade onset. The resultant visual response of the neuron, in this one trial, was $37 \%$ of the average FF visual response before inactivation.

As seen in the mostly overlapping marginal histograms of PSD below the abscissa, summed from the scatter plots above, there was a mild increase in PSD from before to during inactivation (medians 95 vs. 127 ms respectively; 34\% increase; MannWhitney Rank Sum test, $\mathrm{p}<0.001)$. However, the drop in visual responsiveness at the FF 
was far greater than this mild increase in PSD, as seen in the marginal histograms of normalized firing rates to the left of the ordinate, which were highly separated from each other (medians before, 1.00; during, 0.37; 63\% decrease; $\mathrm{p}<0.001$ ).

We then directly compared visual sensitivity in the FF after removing PSD as a factor. To do this we analyzed normalized firing rates only from a selected, discrete range of PSD data, from 50 to $100 \mathrm{~ms}$. Within this range, median PSD was not different before vs. during inactivation (medians 71 vs. 77 ms, Mann-Whitney Rank Sum test, p = 0.2). As shown in the inset, even with PSD matched in this way, the average drop in FF visual sensitivity was 55\% ( $\mathrm{p}<0.001)$, just as calculated in the main text (Fig. 4d, left).

We analyzed the effect of PSD using another method, too. In each experiment we eliminated trials with outlying PSDs using an unbiased, alternating selection algorithm: we removed the shortest PSD trial from the "before" data, then the longest PSD trial from the "during" data, and so forth, until the median PSDs of the two data sets were as close as possible. In each of the 8 experiments we obtained before and during data subsets having median PSDs matched to within 3 ms. Using these PSD-matched data, we found that the deficit at the FF was still severe (same analysis as Fig. 4d, left; 44\% deficit; p = $0.01)$.

In summary, although saccadic reaction times inevitably increase during MD inactivation, this was not a significant confound in our study. Accounting for reaction time changes, the deficit in this study was $\sim 50 \%$ regardless of the analysis method.

A second alternative explanation for our inactivation results is that FEF neurons inherit their shifting RFs in toto from the SC, and MD inactivation simply disrupted that transfer of information. We think that this can be ruled out, however, because shifts in 
the SC are radically different from those in the FEF: in the SC, they never start during a saccade $^{28}$ while in the FEF, they frequently do ${ }^{14,15}$ (Fig. 3c). Finally, a third possibility is that we disrupted corollary discharge arising from MD but which did not originate from the SC; perhaps it came from another area ${ }^{29}$. While we cannot rule this out, we would note that we targeted our inactivation directly onto highly localized, physiologically identified relay neurons of the SC-MD-FEF pathway, that this pathway conveys signals consistent with corollary discharge ${ }^{10}$, that this pathway is necessary for corollary discharge-dependent behavior ${ }^{11}$, and that this is the only known pathway for carrying corollary discharge via thalamus to the cerebral cortex in the monkey brain. Moreover, in previous work we explicitly compared the signals sent from SC to MD with those carried by MD relay neurons, and we found that the SC input almost perfectly explained the MD activity (Ref. 10, see especially Figs. 11A and 13B). While MD relay neurons may get extracollicular input, the evidence to date is that their activity is dominated by SC input. Attributing the observed deficits to the SC-MD-FEF pathway, therefore, stands as the most parsimonious explanation. 


\section{Sommer \& Wurtz}

Figure S1

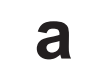

a
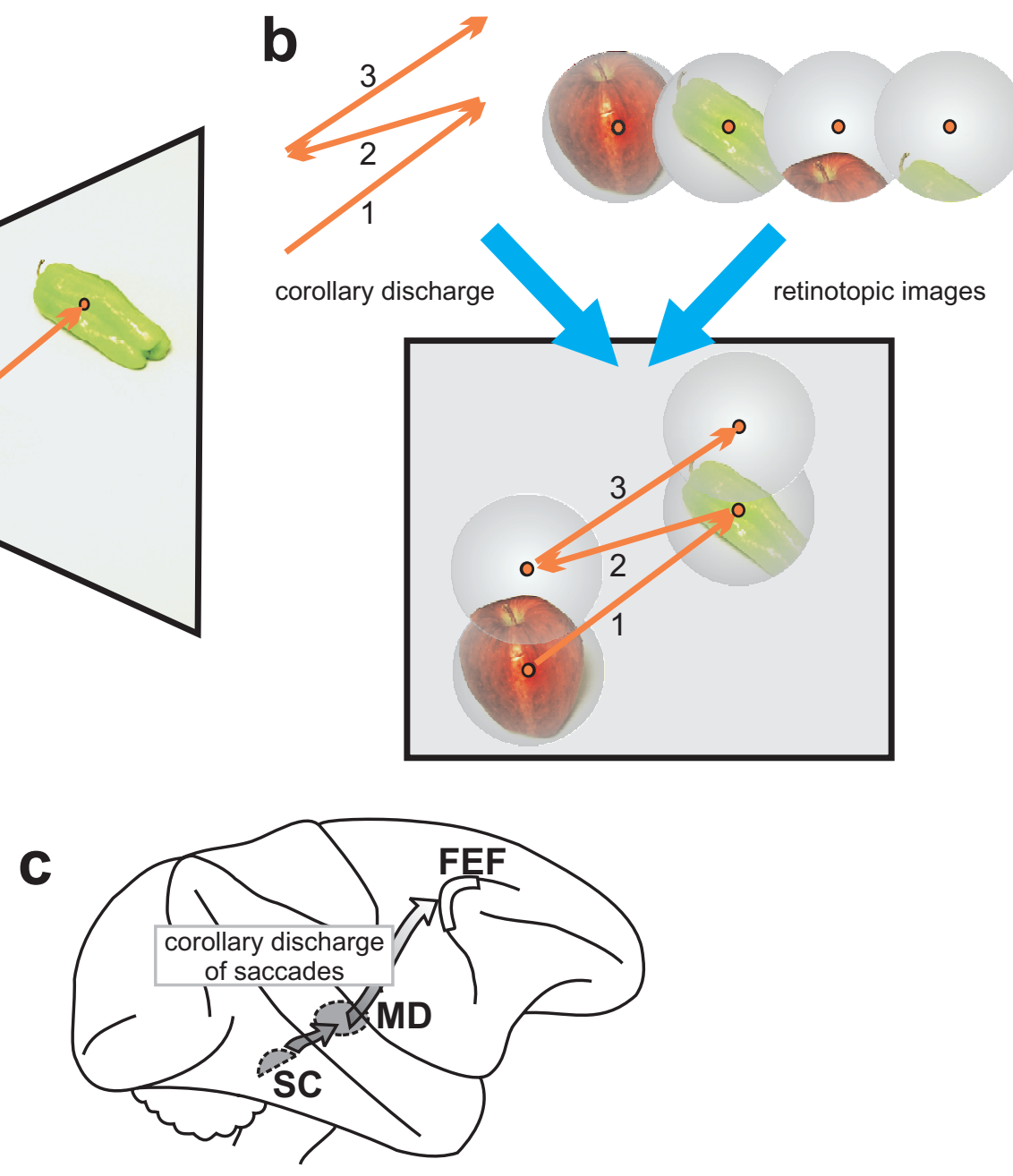

Figure S1: Maintaining a percept of visual stability across saccades. (a) A subject may look at an apple (dotted line) and make a saccade (orange arrow) to a pepper. (b) Several such saccades (arrows 1-3 at top left) will cause sequential images on the retina (top right). The visual system is thought to use corollary discharge to interpret these retinotopic visual changes. A percept of visual stability can be constructed by integrating corollary discharge and retinal images (bottom). (c) We hypothesize that a corollary discharge used for spatial visual analysis rises from superior colliculus (SC) through mediodorsal thalamus (MD) to the frontal eye field (FEF). 


\section{Sommer \& Wurtz \\ Figure S2}

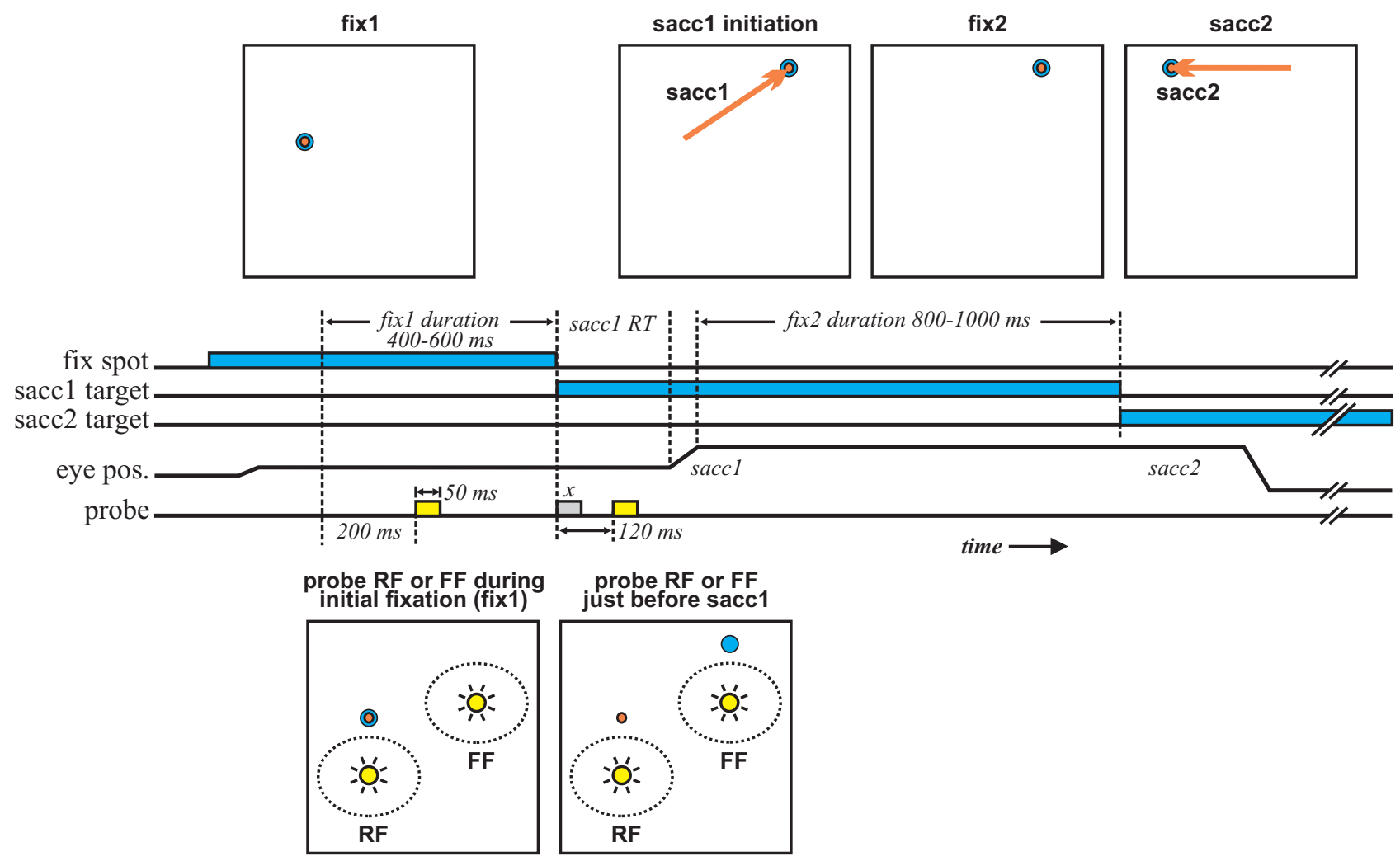

Figure S2: Details of the task. Temporal aspects are indicated by the timeline, and spatial aspects are indicated by rectangular frames showing the RF, FF, probes, fixations, and saccades (as in Fig. 1). Each frame is aligned to the approximate time along the timeline that it occurred. There were 2 fixations (fix 1 and fix 2 ) and 2 saccades (sacc1 and sacc2). Sacc2 was important to discourage saccadic planning toward the FF, but was not relevant to the results and is ignored in the main text. Only one probe was presented per trial, at the RF or FF and during initial fixation or just before sacc1. A trial was omitted from analysis if the saccade began while the probe was still lit. Shown is the spatial configuration for the example neuron of Figure 1. Each neuron had a different RF placement and required a different stimulus arrangement, but the configuration shown was typical. Fix spot, initial fixation spot; sacc1 target, stimulus toward which sacc1 was directed; sacc2 target, stimulus toward which sacc2 was directed; eye pos., eye position; probe, stimulus used to test visual sensitivity; " $x "$, described in Supplementary Methods. 


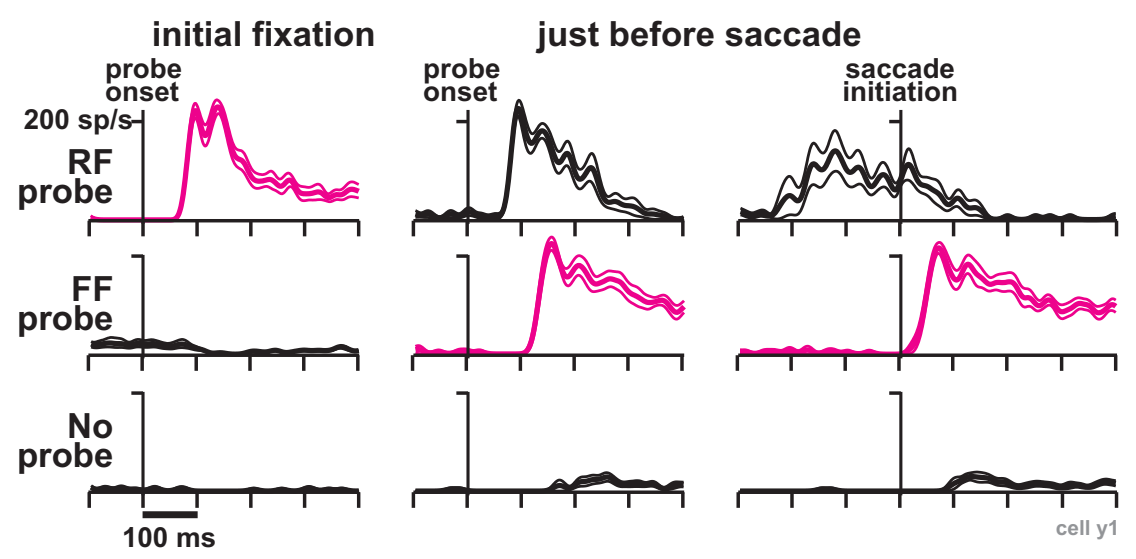

Figure S3: Example of a neuron that became visually responsive at its FF just before the saccade while remaining visually responsive at its RF. This is in contrast to the neuron in Figure $1 \mathrm{c}$ that showed a decrease in visual sensitivity at the RF while the activity at its FF increased. Same format as in Figure 1c. 
a

measurement of edge effects

fixation
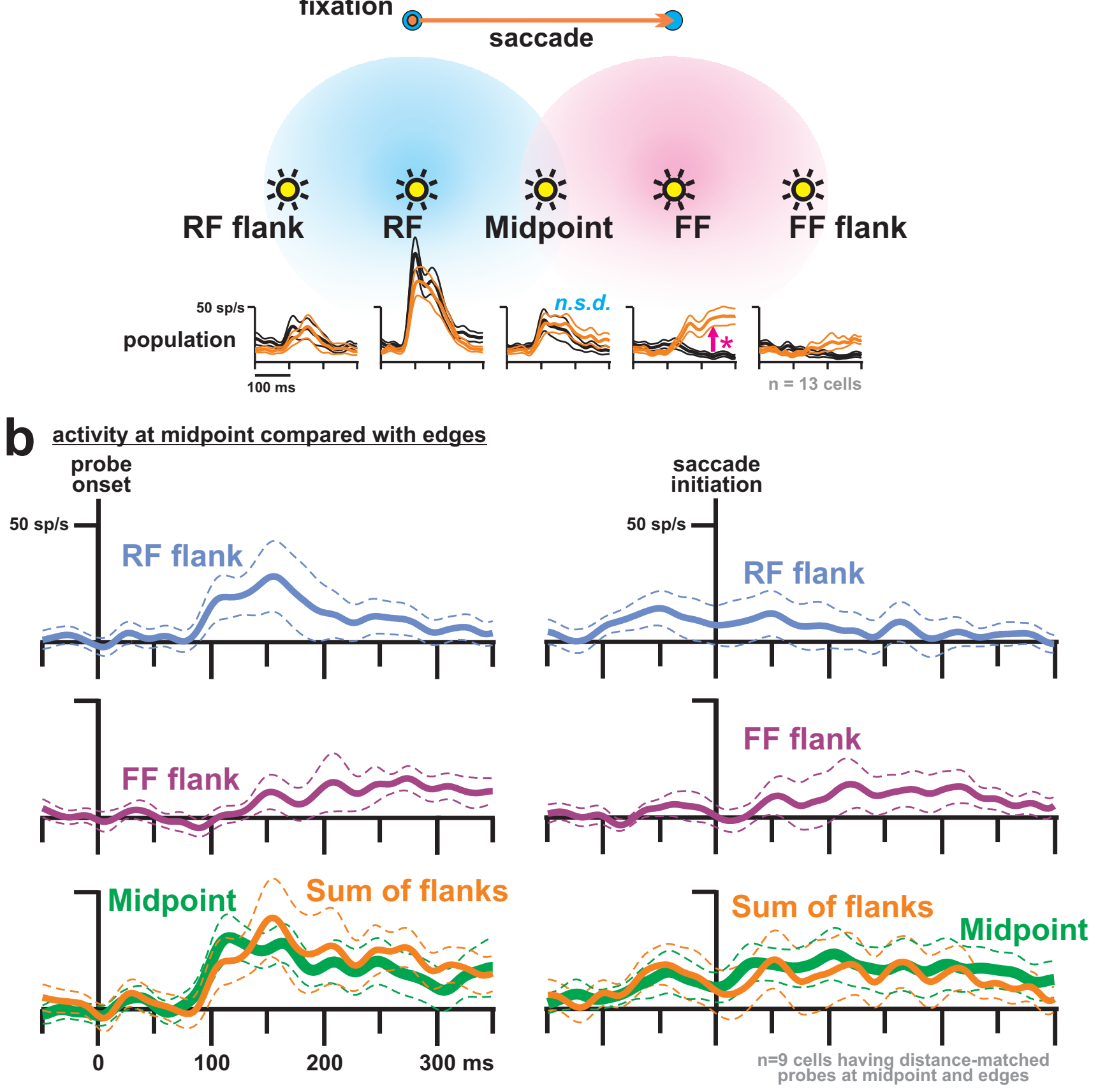

Figure S4: Further spatial analysis, part 1: Effects of response field edges on midpoint activity. (a) Because of large response fields in the FEF, it was usually unavoidable that the midpoint location fell on the edge of the RF, FF, or both, as depicted here. We 


\section{Sommer \& Wurtz Figure S4, continued}

hypothesized that this could account for the low-level midpoint activity in the population data of Fig. 2c (reproduced here in the middle three graphs; for details of data presentation, see Fig. 2c). That is, the midpoint activity likely represented a summation of edge responses from the RF and FF. To test this hypothesis, we quantified each edge response separately using randomly interleaved probes at flanking locations ( $R F$ flank and FF flank). The straightforward assumption was that the response fields were symmetric. Qualitatively, it did appear that summing the signals at the RF flank (leftmost graph) and FF flank (rightmost graph) would explain the midpoint activity (middle graph). (b) To test this observation quantitatively, we studied 9 neurons for which the flank locations and midpoint were exactly equidistant from the RF/FF centers. Average ( \pm SE) RF flank activity is shown in the top row and average FF flank activity in the middle row. The sum of flank activities is compared with the midpoint activity in the bottom row. There is no significant difference at any time, regardless of alignment to probe onset (left) or saccade initiation (right). The small amount of midpoint activity in our data, therefore, is explained by passive summation of RF and FF edge activity. There is no extra activity at the midpoint that can be attributed to an active spread from RF to FF. 


\section{Sommer \& Wurtz \\ Figure S5}
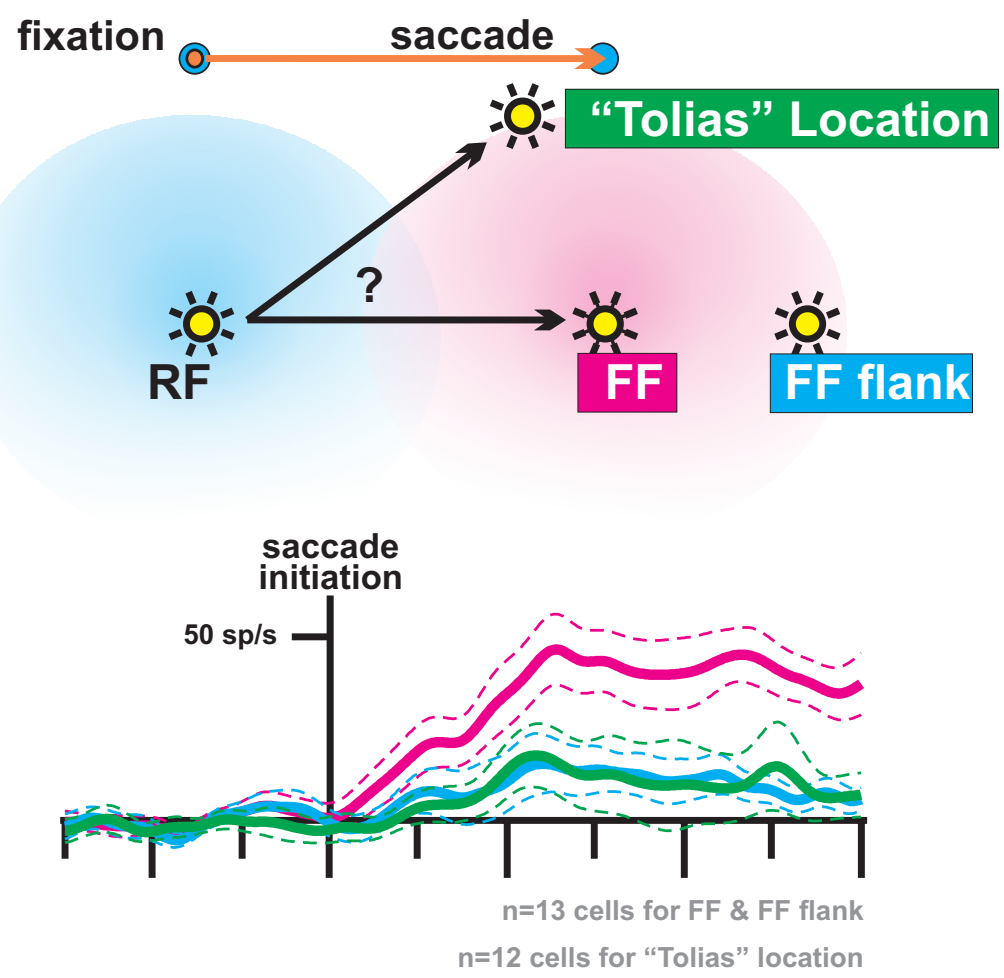

Figure S5: Further spatial analysis, part 2: Test of whether the shift veered toward the saccadic endpoint which would mean our midpoint location was misplaced. At top, the question is schematized: Does the visual response move parallel to the saccade as we presume (to the FF), or toward the saccadic endpoint as found by Tolias et al. ${ }^{30}$ for another cerebral cortical region, visual area V4? For 12 neurons we added a probe near the saccadic target, along a radius toward the RF, as shown ("Tolias" location). The prediction of the Tolias et al. result is that this location would be even more responsive than the FF. Our prediction is that this location would be at the edge of the FF and, if active at all, would be about as active as the FF flank location. As shown below, the probe at the "Tolias" location evoked a visual response (green) identical to that at the FF flank (blue) and much less than that at the FF (magenta). Hence the shift did not veer toward the saccadic endpoint. 


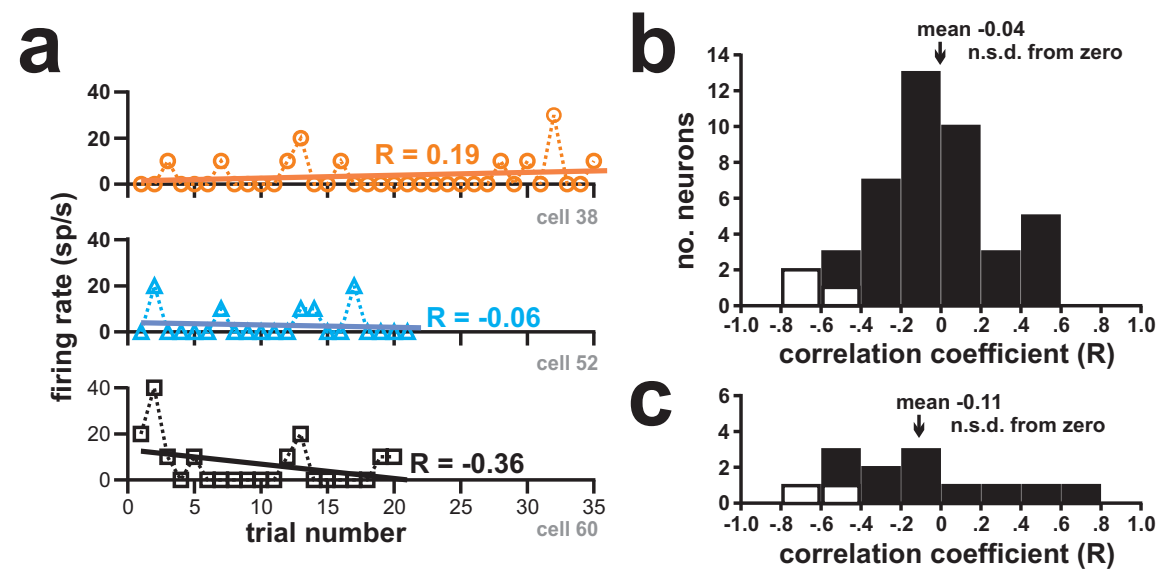

Figure S6: Stability of activity over time. Umeno and Goldberg ${ }^{15}$ reported that shifting RF activity may wax and wane for some neurons. Specifically, they described a memory-like form of shifting RF activity that may emerge in no-probe trials. A hypothesis, therefore, is that activity at the midpoint takes time to grow, emerging only in later trials. (a) First we tested for the basic effect of Umeno and Goldberg. We plotted activity in no-probe trials $(y$-axis) as a function of successive trial number ( $x$-axis). The epoch in which we calculated no-probe firing rate was time-matched, for each neuron, to the epoch used to measure shifts in the FF probe trials. In three example neurons, noprobe activity increased slightly over time (top), changed very little (middle), or decreased slightly (bottom). (b) Overall in our 43 neurons with shifting RFs, the average correlation coefficient was not significantly different from zero. The few neurons with significantly changing activity over time exhibited decreases (white). In sum, activity was very stable during the recording sessions. (c) Result for trials in which we flashed a probe at the midpoint. Correlation coefficients were derived in the same manner as for the no-probe trials (firing rate vs. trial number). Midpoint activity was stable over time on average, but decreased significantly for two neurons. It never increased significantly, refuting the hypothesis that activity builds up at the midpoint during a session. Successive trials are approximately $1 \mathrm{~min}$. apart in these plots. 
Table: summary of all inactivations

\begin{tabular}{|c|c|c|c|c|}
\hline neuron & monkey & $\begin{array}{c}\text { muscimol } \\
\text { injected }(\mu \mathrm{l})\end{array}$ & $\begin{array}{c}\text { deficit in FF } \\
\text { for contra } \\
\text { saccades }\end{array}$ & $\begin{array}{c}\text { presentation } \\
\text { in figures }\end{array}$ \\
\hline 1 & $\mathrm{~T}$ & 1.4 & $16 \%$ & - \\
\hline 2 & $\mathrm{~T}$ & 3.6 & $55 \%^{* *}$ & - \\
\hline 3 & $\mathrm{~T}$ & 1.0 & $55 \%^{*}$ & panel a to right \\
\hline 4 & $\mathrm{Y}$ & 2.0 & $70 \%^{* *}$ & fig. 4b \\
\hline 5 & $\mathrm{Y}$ & 0.4 & $61 \%^{* *}$ & - \\
\hline 6 & $\mathrm{Y}$ & 0.6 & $66 \%^{* *}$ & panel c to right \\
\hline 7 & $\mathrm{Y}$ & 0.4 & $49 \%^{* *}$ & panel b to right \\
\hline 8 & $\mathrm{Y}$ & 0.6 & $57 \%^{* *}$ & - \\
\hline
\end{tabular}
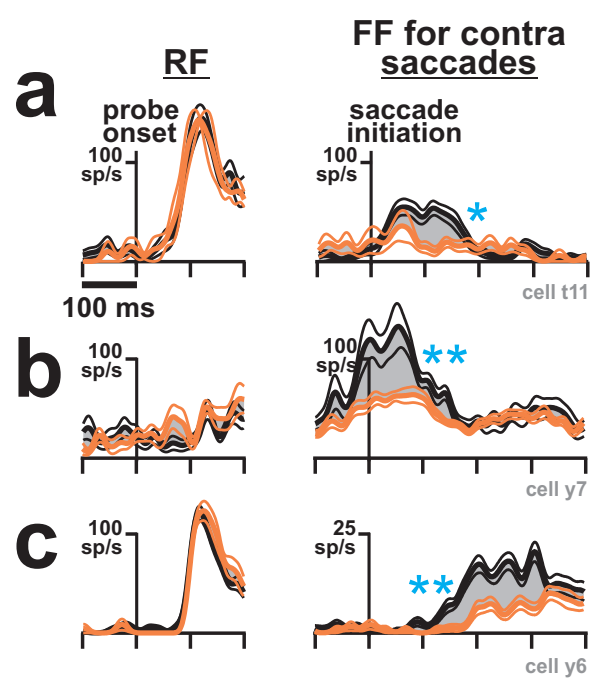

${ }^{*} \mathrm{p}<0.05 ;{ }^{* *} \mathrm{p}<0.001 ;-$, not illustrated

Figure S7: Left: Table showing the details from each inactivation experiment. (a-c)

Illustrations of the deficits for three more neurons. Same format as in Fig. $4 \mathrm{~b}$. 
Deficit is not an artifact of longer saccadic reaction times

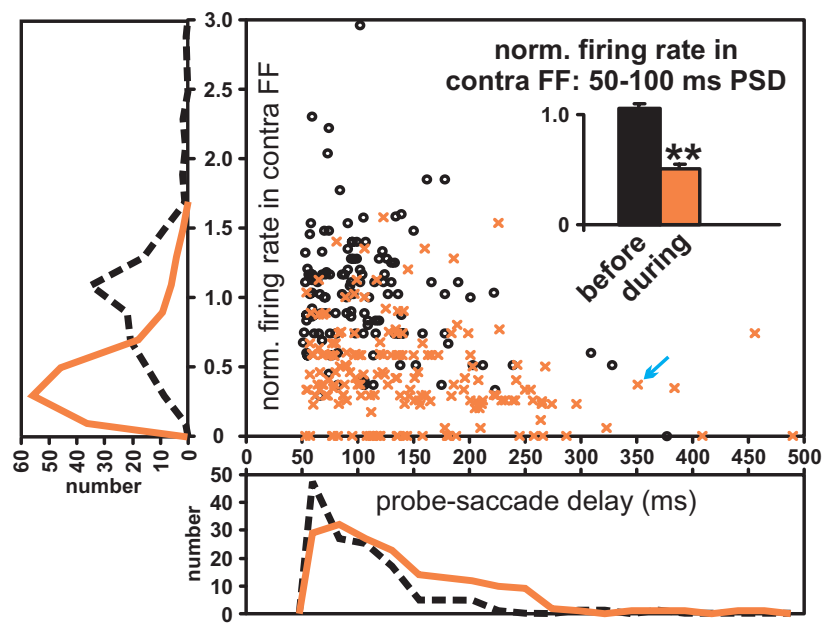

Figure S8: Demonstration that longer reaction times during MD inactivation ${ }^{11}$ had no influence on our results. In the main graph (scatter plot), data from every individual trial of the 8 inactivation experiments are plotted as described in the Supplementary Discussion; the blue arrow is discussed in that section. Left histogram shows distribution of normalized (norm.) firing rates in the FF, derived from the scatter plot. Bottom histogram shows distribution of probe-saccade delays (PSDs). Inset shows the average and SE of norm. firing in the FF during the PSD interval 50-100 ms. Only the FFs that accompanied contraversive saccades are considered. As in main text, black represents before inactivation and orange during inactivation. ${ }^{* *}, \mathrm{p}<0.0001$. 


\section{Abstractions}

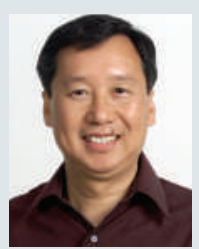

\section{LEAD AUTHOR}

Since the early 1990 s, physicist Steven Louie at the University of California, Berkeley, has been probing the useful properties of carbonbased nanomaterials. On page 347 , Louie and his co-workers present a theoretical prediction of some potentially intriguing properties of nanoscale ribbons of graphene. The team suggests that the nonmetallic graphene could be induced to take on some characteristics of a metal, such as magnetism. The result would be a 'half-metal' that could potentially be used in spintronics - a refined version of electronics that makes use of not only the electrons' charge but also their 'spin'. The calculations might pave the way for fresh approaches to spintronic devices using graphene nanoribbons. Louie spoke to Nature about his work.

How can a non-metallic element such as carbon have metal properties?

A graphene ribbon has a unique geometry in that it has zigzag edges. Low-energy electrons, with their spins aligned either up or down, are able to run freely along these edges. It is the edge electrons that give rise to magnetism. As the magnetic states are spatially separated across the ribbon, you can use a transverse electric field to shift their energy, thereby creating charge carriers with the same spin, making the system a half-metal. Another electric field along the ribbon length can then be used to drive a spin current.

What is holding back exploration of these properties in organic materials?

First, half-metals are very rare, and organic materials tend not to be magnetic. Typically, electrons in organic materials form covalent bonds - the spin up and spin down are paired in the bond so there is no net spin - or the spins are randomly oriented. But in a zigzag graphene nanoribbon, the atoms on the edge behave differently, forming edge states capable of having a net spin. You wouldn't find this behaviour in a perfect buckyball or a nice, long carbon nanotube.

Why would organic materials be advantageous in electronics?

The driving force for wanting organic materials is that they are composed of abundant and non-toxic elements. It would be great to have cheap, high-performance electronics that are less harmful to the environment.

Will spintronics replace electronics? I don't know whether spintronics will eventually replace electronics, but it will enhance today's electronics. The challenge now is to create the right material to efficiently generate spin current.
MAKING THE PAPER

Marc Sommer

\section{Tracking down the signal that provides us with seamless vision.}

As we look at the world around us, our eyes are constantly on the move. But, somehow, what we see is not a series of disjointed, disconnected still pictures but a seamless film. How our brain manages to compensate for our rapid eye movements has puzzled neuroscientists for many years. On page 374, Marc Sommer and Robert Wurtz offer evidence that may help solve the mystery.

Wurtz, a neuroscientist at the US National Eye Institute in Bethesda, Maryland, had tried and failed to resolve the question back in 1968. But when Sommer joined his lab in 1998, a surprising result in a related project led them back to the problem - and offered them a solution.

The pair had been using monkeys to examine signals that travel from part of the brain's cortex that processes visual stimuli to an area of the brainstem linked with eye movement. Every now and then they saw something strange happen: a signal went in the opposite direction. A neuron in the brainstem fired, causing a response in the cortex. "The brainstem was talking back to the cortex," says Sommer.

This result puzzled the two researchers, until they realized that they might be looking at the pathway that eluded Wurtz all those years ago. Maybe the brainstem was sending a signal to the cortex to alert it to an upcoming eye movement. Based on anatomical layout, they suspected that the signal was passing through 'relay' neurons in the thalamus on its way to the cortex. A series of preliminary experiments suggested that they were right, and the thalamus was involved. But going on to prove that the whole signal pathway existed was no easy task.

The pair set up a conceptually simple, if technically difficult, experiment. They used three probes implanted into the brain of a monkey:

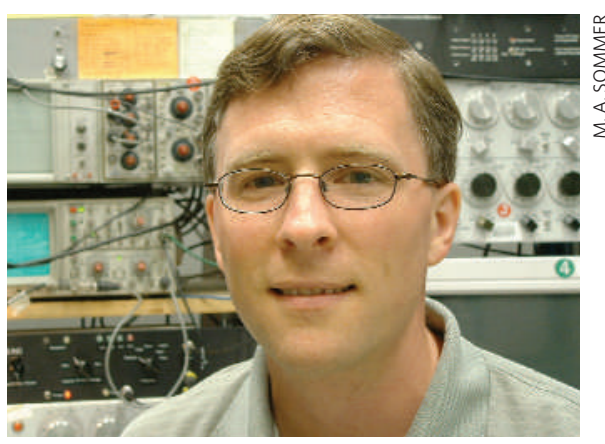

one to stimulate nerve cells in the brainstem, one to switch off neurons in the thalamus, and one to record the activity of a neuron in the cortex. "These are fundamental techniques of neurophysiology, but the challenge was to do all three at the same time in an awake animal," says Sommer.

To get his measurements, Sommer had to painstakingly identify a single cell in the cortex that he could monitor. He then had to record its activity for three hours in a live, moving animal. As if all this was not difficult enough, Sommer had by this time begun to set up his own laboratory at the University of Pittsburgh. "I would fly back at weekends hoping to get one more neuron," he recalls.

It took six months to record signals from eight neurons. But that was enough to show that the pathway exists. The brainstem seems to send a signal via the thalamus to the cortex to warn it of an impending eye movement. In response, neurons in the cortex adjust the position of the field of vision to where the eye will move next. When Sommer used the probe in the thalamus to switch off the relay neurons, the signal didn't get through and the cortex didn't take any steps to compensate for eye movement.

Now, Sommer and Wurtz want to find out what effect blocking this signal has on vision. Will the monkeys end up seeing the world as a series of disjointed snapshots? Sommer is currently devising tests to answer this question - but he first needs to find a way to measure what the monkeys are actually perceiving.

\section{KEY COLLABORATION}

The seeds for the collaboration behind the paper on page 354 of this issue were sown some 20 years ago, while Miquel Canals was doing his PhD at the University of Perpignan in France. Back then he was studying the Gulf of Lions in the northwest Mediterranean Sea, mapping the region with what he now describes as "rudimentary tools".

Over the intervening years, Canals, now at the University of Barcelona in Spain, and his colleagues at Perpignan have revisited the area, refining their measurements using more sophisticated equipment. By pooling their limited resources - including a number of small grants - they have managed to investigate the mechanisms by which sediment and organic matter are flushed from shallow to deep water through submarine canyons.

"None of the groups had enough equipment to do it by themselves," Canals says. They found that the movement of sediment could be triggered by a form of current that is driven by sea density. Their measurements of the effects of this current, the sediment movement, the changes to the ocean floor, and the presence of deep-water coral, have already led to a large grant from the European Union, which should keep the groups occupied in the gulf for quite a few years to come. 